\title{
Nonlinear Wave-Current Interaction in Water of Finite Depth
}

\author{
Zhen Liu ${ }^{1}$, Zhiliang Lin $^{2}$, Longbin $\mathrm{Tao}^{3}$, Jian Lan $^{4}$
}

\section{Abstract:}

The interaction of nonlinear progressive waves and a uniform current in water of finite depth is investigated analytically by means of the homotopy analysis method (HAM). With HAM, the velocity potential of the flow and the surface elevation are expressed by Fourier series and the nonlinear free surface boundary conditions are satisfied by continuous mapping. Unlike a perturbation method, the present approach does not depend on any small parameters; thus the solutions are suitable for steep waves and strong currents. To verify the HAM solutions, experiments are conducted in the wave-current flume of The Education Ministry Key Laboratory of Hydrodynamics at Shanghai Jiao Tong University (SJTU). It is found that the HAM solutions are in good agreement with experimental measurements. Based on the series solutions of the validated analytical model, the influence of water depth, wave steepness and current velocity on the physical properties of the coexisting wave-current field are studied in detail. The variation mechanisms of wave characteristics due to wave-current interaction are further discussed in a quantitative manner. The significant advantage of HAM in dealing with strong nonlinear wave-current interactions in the present study is clearly demonstrated in which the solution technique is independent of small parameters. A comparative study on

${ }^{1}$ Zhen Liu, PhD student, State Key Laboratory of Ocean Engineering, Shanghai Jiao Tong University, 800 Dongchuan Road, Shanghai 200240, China. E-mail: liuzhen0829@sjtu.edu.cn

2Zhiliang Lin, Associate Professor, State Key Laboratory of Ocean Engineering, Collaborative Innovation Center for Advanced Ship and Deep-Sea Exploration(CISSE), Shanghai Jiao Tong University, 800 Dongchuan Road, Shanghai 200240, China. E-mail: linzhiliang@sjtu.edu.cn

${ }^{3}$ Longbin Tao, Professor (M.ASCE), School of Marine Science and Technology, Newcastle University, Armstrong Building, Newcastle upon Tyne NE1 7RU, United Kingdom (corresponding author). E-mail: longbin.tao@newcastle.ac.uk

${ }^{4}$ Jian Lan, Master's student, State Key Laboratory of Ocean Engineering, Shanghai Jiao Tong University, 800 Dongchuan Road, Shanghai 200240, China. E-mail: lanjian2013@sjtu.edu.cn 
wave characteristics further reveals the great potential of HAM to solve more complex wavecurrent interaction problems leading to engineering applications in the offshore industry and the marine renewable energy sector.

Keywords: Wave-current interaction; Nonlinear; Finite water depth; Homotopy analysis method

\section{Introduction}

The co-existence of waves and currents is a common feature of most marine environments. Nonlinear wave-current interaction is an important topic in both coastal and offshore engineering. Giant waves (freak waves) have been registered in many regions of the oceans, especially off the east coast of South Africa, where strong interactions between waves and opposing currents exist (Mallory 1974; Kharif and Pelinovsky 2003). In these cases, the opposing current significantly augments the wave height and steepness, resulting in considerable hazards for ships and offshore structures. During the past several decades, wavecurrent interaction has been the subject of numerous research efforts. Most of them are well documented in the review articles by Peregrine (1976), Jonsson (1990) as well as Thomas and Klopman (1997).

In many practical instances the current velocity varies significantly with depth, leading to the creation of a velocity profile, for example, with a wind-driven current where the magnitude of the current velocity varies exponentially with depth. Studies based on this type of current have been reported in the literature (Thomas 1981; Thomas 1990; Swan et al. 2001; Swan and James 2001). In some other cases, however, it is reasonable to assume that the current velocity is 
approximately uniform with depth. Examples of this type of current include large scale ocean currents, and the majority of tidal flows where the time and length scales over which the current varies are much larger than the wave period or wavelength. Rienecker and Fenton (1981) presented the numerical solution for steady water waves progressing in constant water depth based on the Fourier approximation method. In their model, the time mean Eulerian velocity, i.e. the current velocity, can be taken into account. Later, this method is further simplified by Fenton (1988) and applied to waves in both deep and shallow water conditions. Fenton (1985) proposed a $5^{\text {th }}$-order perturbation solution for waves propagating on a uniform current in constant water depth. For not-too-high waves and not-too-shallow water depths, the analytic solution given by Fenton (1985) was in good agreement with the numerical solution by Rienecker and Fenton (1981). However, it is worth noting that the perturbation solution procedure by Fenton (1985) is rather complicated and difficult to extend to solve the more complex interaction of multiple waves and a current. Umeyama (2011) also reported a $3^{\text {rd }}$-order perturbation solution and experimental data for waves propagating on a following current. It is important to point out that the experimental conditions in his work possess relatively weak nonlinearity and low current velocities. Based on a Lagrangian coordinate system, Chen and Chen (2014) also obtained a $5^{\text {th }}$-order perturbation series approximation for the interaction of progressive waves and uniform currents. The focus of their research is on the wavy track of the particle motion. Though there are several theoretical works on waves propagating on favorable or adverse uniform currents, few analytical models describing the interaction between steep waves and strong currents, as well as the effect of water depth, can be found in the literature. 
development. Different from the perturbation method, HAM does not depend on any small parameter, so it is suitable for solving strong nonlinear problems. HAM was first applied to water waves in infinite water depth by Liao and Cheung (2003). Later, Tao et al. (2007) successfully extended Liao and Cheung (2003) to water of finite depth. Xu (2006) applied HAM to investigate nonlinear wave and uniform current interaction in infinite water depths. It was shown that the phase velocity of the waves in deep water obtained by HAM agrees well with experimental measurements. In the framework of HAM, Cheng et al. (2009) investigated the interaction of deep water waves and exponential shear currents. Liu et al. (2014) considered the phase velocity effects of bi-chromatic wave interaction with exponentially sheared currents by means of HAM. Examples can also be found in the literature demonstrating the effectiveness of HAM to solve more complicated wave-wave interaction problems (Liao 2011; Xu et al. 2012; Liu and Liao 2014; Lin et al. 2014).

The objective of the present study is to investigate the interaction between steep waves and strong uniform currents in water of constant finite depth by HAM. In contrast to a perturbation solution, the HAM series solution is independent of small parameters and thus possesses considerable accuracy for strongly nonlinear problems. By including constant water depth in the solution procedure, the present work further investigates the influence of water depth on the nonlinear wave-current interaction problem in detail due to its significance in the shallow water coastal region. To validate the effectiveness of the present approach, experiments are conducted and the data are used to compare with the present HAM solution. The present paper is organized as follows. The following section provides a description of governing equations and boundary conditions; HAM is presented for a wave-current interaction problem; and the detailed solution 
techniques are discussed. Following this section, the experimental setup and measurement techniques are described. Finally, detailed analytical results about how opposing currents and water depths influence the wave parameters of wave-current coexisting fields are presented.

\section{Theoretical Consideration}

\section{Governing Equations and Boundary Conditions}

\section{The Description of Wave-Current Interaction}

Consider the interaction between two-dimensional, nonlinear, progressive waves and a uniform current in water of finite depth. The fluid is assumed to be inviscid and incompressible, and the flow is irrotational. A Cartesian coordinate system $(x, z)$ is adopted where the $\mathrm{x}$-axis is positive in the direction of wave propagation, and the z-axis is positive vertically upwards from the still water level as shown in Fig. 1. The quantities $\varphi^{*}(x, z, t)$ and $\zeta(x, t)$ are defined as the velocity potential and the wave elevation, respectively. The fluid motion described by the velocity potential $\varphi^{*}(x, z, t)$ is governed by the Laplace equation:

$$
\nabla^{2} \varphi^{*}(x, z, t)=0, \quad-\infty<x<+\infty,-d<z<\zeta(x, t)
$$

and subject to two free surface boundary conditions:

$$
\begin{gathered}
\frac{\partial \zeta}{\partial t}+\frac{\partial \varphi^{*}}{\partial x} \frac{\partial \zeta}{\partial x}-\frac{\partial \varphi^{*}}{\partial z}=0, \quad z=\zeta(x, t) \\
g \zeta+\frac{1}{2}\left(\nabla \varphi^{*}\right) \cdot\left(\nabla \varphi^{*}\right)+\frac{\partial \varphi^{*}}{\partial t}=\frac{1}{2} U_{0}^{2}, \quad z=\zeta(x, t)
\end{gathered}
$$

and the following condition at the bottom:

$$
\frac{\partial \varphi^{*}}{\partial z}=0, \quad z=-d
$$

where $\nabla=(\partial / \partial x, \partial / \partial z), t$ denotes time, $g$ is gravitational acceleration, $d$ is the water depth and $U_{0}$ is the uniform current velocity. Since gravity capillary waves caused by surface tension are quite small compared to their wavelengths, the effect of surface tension is neglected. By means of superposition for potential theory, the total velocity potential of the wave-current co-existing field is given by $\varphi^{*}=U_{0} x+\varphi$, where $\varphi$ denotes the wave velocity potential. 


$$
\frac{\partial^{2} \varphi^{*}}{\partial t^{2}}+g \frac{\partial \varphi^{*}}{\partial z}+\frac{\partial\left[\left(\nabla \varphi^{*}\right) \cdot\left(\nabla \varphi^{*}\right)\right]}{\partial t}+\frac{1}{2}\left(\nabla \varphi^{*}\right) \cdot \nabla\left[\left(\nabla \varphi^{*}\right) \cdot\left(\nabla \varphi^{*}\right)\right]=0, \quad z=\zeta(x, t)
$$

Substituting $\varphi^{*}=U_{0} x+\varphi$ into Eqs. (1), (3), (4) and (5), the governing equation becomes:

$$
\nabla^{2} \varphi(x, z, t)=0, \quad-\infty<x<+\infty,-d<z<\zeta(x, t)
$$

which is subject to two nonlinear free surface conditions:

$$
\begin{gathered}
g \zeta+U_{0} \frac{\partial \varphi}{\partial x}+\frac{1}{2} \nabla \varphi \cdot \nabla \varphi+\frac{\partial \varphi}{\partial t}=0, \quad z=\zeta(x, t) \\
\frac{\partial^{2} \varphi}{\partial t^{2}}+g \frac{\partial \varphi}{\partial z}+2 U_{0} \frac{\partial^{2} \varphi}{\partial x \partial t}+\frac{\partial(\nabla \varphi \cdot \nabla \varphi)}{\partial t}+U_{0}{ }^{2} \frac{\partial^{2} \varphi}{\partial x^{2}} \\
+U_{0} \frac{\partial(\nabla \varphi \cdot \nabla \varphi)}{\partial x}+\frac{1}{2} \nabla \varphi \cdot \nabla(\nabla \varphi \cdot \nabla \varphi)=0, \quad z=\zeta(x, t)
\end{gathered}
$$

and the following bottom boundary condition:

$$
\frac{\partial \varphi}{\partial z}=0, \quad z=-d
$$

\section{Variable Transformation}

The objective of this paper is to study the interaction between nonlinear progressive waves and a uniform current in an arbitrary, uniform water depth. Without loss of generality, assume that the wave-current co-existing field is made up of a current and a wave component with wave number $k$ and corresponding angular frequency $\omega$. It is convenient to define the phase function

$$
\theta=k x-\omega t+\theta_{0}
$$

where $\theta_{0}$ denotes an arbitrary, constant phase for zero time at the origin of the $(x, z)$ coordinate system. The above variable can be used to replace the variables $x$ and $t$, and then the time, $t$, will not appear explicitly for a steady wave-current system. Thus, one can express the potential function $\varphi(x, z, t)=\phi(\theta, z)$, and the wave elevation $\zeta(x, t)=\eta(\theta)$ for the coexisting field of one train of progressive waves and a uniform current. With these definitions, 
140

\section{7 where}

148

$$
\begin{aligned}
\omega^{2} \frac{\partial^{2} \phi}{\partial \theta^{2}}+g \frac{\partial \phi}{\partial z}-2 \omega \frac{\partial f}{\partial \theta} & +\hat{\nabla} \phi \cdot \hat{\nabla} f-2 U_{0} k \omega \frac{\partial^{2} \phi}{\partial \theta^{2}} \\
& +U_{0}{ }^{2} k^{2} \frac{\partial^{2} \phi}{\partial \theta^{2}}+2 U_{0} k \frac{\partial f}{\partial \theta}=0, \quad z=\eta(\theta)
\end{aligned}
$$

151 and $\hat{\nabla}=(k \partial / \partial \theta, \partial / \partial z)$.

\section{HAM for the Wave-Current Interaction}

153

$$
\left\{\sin (m \theta) \frac{\cosh [m k(z+d)]}{\cosh (m k d)} \mid m \geq 1\right\}
$$

in the form:

$$
\phi(\theta, z)=\sum_{m=1}^{+\infty} b_{m} \Psi_{m}(\theta, z)
$$


where

$$
\Psi_{m}(\theta, z)=\sin (m \theta) \frac{\cosh [m k(z+d)]}{\cosh (m k d)}
$$

161 and $b_{m}(m=1,2, \cdots)$ are coefficients. This provides us with a rule for the solution expression for $\phi(\theta, z)$ (Liao, 2003). It should be noted that Eq. (19) automatically satisfies the governing equation (11) and the bottom boundary condition (12). Accordingly, the wave elevation can be expressed by a set of base functions:

$$
\{\cos (m \theta) \mid m \geq 1\}
$$

in the form:

$$
\eta(\theta)=\sum_{m=1}^{+\infty} a_{m} \cos (m \theta)
$$

where $a_{m}$ are coefficients to be determined.

\section{Zeroth-Order Deformation Equation}

In the framework of HAM (Liao, 2003), there is great freedom to choose the linear auxiliary operator. According to the linear part of the nonlinear boundary conditions (13) and (14), two linear auxiliary operators are chosen as:

$$
\begin{gathered}
\mathrm{L}_{1}[(\cdot)]=(\cdot) \\
\mathrm{L}_{2}[\phi]=\bar{\omega}^{2} \frac{\partial^{2} \phi}{\partial \theta^{2}}+g \frac{\partial \phi}{\partial \mathrm{z}}
\end{gathered}
$$

where

$$
\bar{\omega}=\sqrt{g k \tanh (k d)}
$$

Based on the nonlinear boundary conditions, two nonlinear operators can be defined as:

$$
\mathrm{N}_{1}[\eta, \phi, \omega]=\eta-\frac{1}{g}\left[\left(\omega-U_{0} k\right) \frac{\partial \phi}{\partial \theta}-f\right]
$$




$$
\begin{aligned}
\mathrm{N}_{2}[\phi, \omega] & =\omega^{2} \frac{\partial^{2} \phi}{\partial \theta^{2}}+g \frac{\partial \phi}{\partial z}-2 \omega \frac{\partial f}{\partial \theta}+\hat{\nabla} \phi \cdot \hat{\nabla} f \\
& -2 U_{0} k \omega \frac{\partial^{2} \phi}{\partial \theta^{2}}+U_{0}{ }^{2} k^{2} \frac{\partial^{2} \phi}{\partial \theta^{2}}+2 U_{0} k \frac{\partial f}{\partial \theta}
\end{aligned}
$$

180 Then the zeroth-order deformation equation can be constructed as:

$$
\hat{\nabla}^{2} \breve{\phi}(\theta, z ; q)=0, \quad-d<z \leq \breve{\eta}(\theta ; q)
$$

182

183

which subject to the bottom boundary condition:

$$
\frac{\partial \breve{\phi}(\theta, z ; q)}{\partial z}=0, \quad z=-d
$$

and the two nonlinear boundary conditions on $z=\breve{\eta}(\theta ; q)$ :

$$
\begin{gathered}
(1-q) \mathrm{L}_{1}[\breve{\eta}(\theta ; q)]=q c_{0} \mathrm{~N}_{1}[\breve{\eta}(\theta ; q), \breve{\phi}(\theta, z ; q), \breve{\omega}(q)] \\
(1-q) \mathrm{L}_{2}\left[\breve{\phi}(\theta, z ; q)-\phi_{0}(\theta, z)\right]=q c_{0} \mathrm{~N}_{2}[\breve{\phi}(\theta, z ; q), \breve{\omega}(q)]
\end{gathered}
$$

where $q \in[0,1]$ is an embedding parameter; $c_{0}$ is the so-called nonzero convergence-control parameter; $\phi_{0}(\theta, z)$ is the initial estimate of the potential function; and $\breve{\phi}(\theta, z ; q), \breve{\eta}(\theta ; q)$ and $\breve{\omega}(q)$ are the mapping functions, respectively.

When $q=0$, the zeroth-order deformation Eqs. (28)-(31) have the solution:

$$
\breve{\phi}(\theta, z ; 0)=\phi_{0}(\theta, z)
$$

$$
\breve{\eta}(\theta ; 0)=0
$$

When $q=1$, the zeroth-order deformation Eqs. (28)-(31) are equivalent to the original Partial Differential Equations (PDEs) (11)-(14), respectively, provided that:

$$
\begin{gathered}
\breve{\phi}(\theta, z ; 1)=\phi(\theta, z) \\
\breve{\eta}(\theta ; 1)=\eta(\theta) \\
\breve{\omega}(1)=\omega
\end{gathered}
$$

Thus, as the embedding parameter $q$ increases from 0 to $1, \breve{\phi}(\theta, z ; q)$ and $\breve{\eta}(\theta ; q)$ deform continuously from initial estimates $\phi_{0}(\theta, z)$ and 0 to become the exact solutions of the original 

frequency $\omega$. parameter $q$, read as:

$$
\breve{\phi}(\theta, z ; q)=\sum_{m=0}^{+\infty} \phi_{m}(\theta, z) q^{m}
$$

$$
\breve{\eta}(\theta ; q)=\sum_{m=0}^{+\infty} \eta_{m}(\theta) q^{m}
$$

$$
\breve{\omega}(q)=\sum_{m=0}^{+\infty} \omega_{m} q^{m}
$$

where

$$
\phi_{m}(\theta, z)=\left.\frac{1}{m !} \frac{\partial^{m} \breve{\phi}(\theta, z ; q)}{\partial q^{m}}\right|_{q=0}
$$

Assuming that $c_{0}$ is properly chosen so that the Maclaurin series (37), (38) and (39) converge at $q=1$, then the so-called homotopy-series solutions are obtained as:

$$
\eta_{m}(\theta)=\left.\frac{1}{m !} \frac{\partial^{m} \breve{\eta}(\theta ; q)}{\partial q^{m}}\right|_{q=0}
$$

$$
\omega_{m}=\left.\frac{1}{m !} \frac{\partial^{m} \breve{\omega}(q)}{\partial q^{m}}\right|_{q=0}
$$

$$
\phi(\theta, z)=\phi_{0}(\theta, z)+\sum_{m=1}^{+\infty} \phi_{m}(\theta, z)
$$

$$
\eta(\theta)=\sum_{m=1}^{+\infty} \eta_{m}(\theta)
$$

$$
\omega=\omega_{0}+\sum_{m=1}^{+\infty} \omega_{m}
$$


equating the like-power of $q$, the so-called $m$ th-order deformation equations are:

where

$$
\eta_{m}(\theta)=\mathrm{R}_{m}^{\eta}\left(\theta ; c_{0}\right)
$$

$\overline{\mathrm{L}_{2}}[\phi]=\left.\mathrm{L}_{2}[\phi]\right|_{z=0}$ and $m \geq 1$. The definitions of $S_{m}, \bar{S}_{m}, \chi_{m}, \Lambda_{m}, \bar{\phi}_{z, m}^{0}, \Gamma_{m, i}$, $\bar{\phi}_{m}^{i} \quad(i=0,1,2)$ and their detailed derivations can be found in Liao (2011).

\section{The Initial Estimate}

Without loss of generality, assume that the wave number $k$ and the coefficient $a_{1}$ in the wave profile of Eq. (22) are given in the wave-current system. The wave frequency $\omega$ is unknown and to be calculated. Liao (2003) has demonstrated that there is great freedom to choose the initial estimate in HAM. Based on the dispersion relation of the linear wave-current interaction, the initial estimate of the frequency $\omega$ is set as: $\omega_{0}=(1+\varepsilon)\left(\bar{\omega}+U_{0} k\right)$, where $\varepsilon$ is a very small, positive real value. The rest of this subsection considers the choice of the initial estimate for $\phi(\theta, z)$. 


$$
\mathrm{L}_{2}\left[\Psi_{m}\right]=\lambda_{m} \cdot \Psi_{m}
$$

240 where $\Psi_{m}$ is defined by Eq. (20) and

$$
\lambda_{m}=g|m k| \tanh (|m k| d)-(m \bar{\omega})^{2}
$$

242 Therefore, the inverse operator $\mathrm{L}_{2}^{-1}$ is defined as

$$
\mathrm{L}_{2}^{-1}\left[\Psi_{m}\right]=\frac{\Psi_{m}}{\lambda_{m}}, \quad \lambda_{m} \neq 0
$$

Note that the inverse operator $\mathrm{L}_{2}^{-1}$ has definition only for non-zero values of $\lambda_{m}$. When $\lambda_{m}=0$, therefore,

$$
g|m k| \tanh (|m k| d)=(m \bar{\omega})^{2}
$$

$$
\phi_{0}(\theta, z)=b_{1} \cdot \Psi_{1}(\theta, z)
$$

where $b_{1}$ is an unknown constant to be determined later. the property of the auxiliary linear operator,

$$
\mathrm{R}_{m}^{\varphi}=\sum_{i=1}^{I_{m}} \tilde{b}_{m, i} \sin (i \theta)
$$

where $\tilde{b}_{m, i}$ are coefficients and $I_{m}$ is related to the right-hand side of Eq. (48). According to has to be enforced to avoid the so-called secular terms. Therefore, using Eq. (57), it is

$$
\tilde{b}_{m, 1}=0
$$
convenient to obtain the solution of Eq. (48): 


$$
\phi_{m}(\theta, z)=\sum_{i=2}^{I_{m}} \bar{b}_{m, i} \Psi_{i}(\theta, z)+\bar{b}_{m, 1} \Psi_{1}(\theta, z)
$$

260

261

where $\bar{b}_{m, 1}$ is an unknown coefficient to be determined in the $(m+1)$ th-order deformation equation. Similarly, according to Eq. (49), there is

$$
\eta_{m}(\theta)=\sum_{i=1}^{I_{m}} \bar{a}_{m, i} \cos (i \theta)
$$

When $m=1$ using Eq. (60), the unknown coefficient $b_{1}$ in Eq. (58) can be obtained for the initial estimate $\phi_{0}(\theta, z)$. When $m \geq 2$, since the coefficient of the primary wave component is given, there are

$$
\sum_{n=1}^{m} \bar{a}_{n, 1}=a_{1}
$$

Thus, Eqs. (60) and (63) provide a set of algebraic equations for $\bar{b}_{m-1,1}$ and $\omega_{m-1}(m \geq 2)$ and make the problem closed. The high-order deformation equations can be solved by means of the symbolic computation software-Mathematica 7. At the $M^{\text {th }}$ - order approximations, we have:

$$
\left\{\begin{array}{l}
\phi(\theta, z) \approx \phi_{0}(\theta, z)+\sum_{m=1}^{M} \phi_{m}(\theta, z) \\
\eta(\theta) \approx \sum_{m=1}^{M} \eta_{m}(\theta) \\
\omega \approx \omega_{0}+\sum_{m=1}^{M} \omega_{m}
\end{array}\right.
$$

\section{Optimal Convergence-Control Parameters}

For the $m^{\text {th }}$-order approximations $\phi(\theta, z)$ and $\eta(\theta)$, there is still one unknown parameter $c_{0}$, which is used to guarantee the convergence of the approximation series. In order to choose an optimal $c_{0}$, two averaged residual square errors of the boundary conditions are defined as: 


$$
\mathrm{E}_{m}^{\eta}=\frac{1}{\left(1+I_{k}\right)} \sum_{i=0}^{I_{k}}\left(\left.\mathrm{~N}_{1}[\phi(\theta, z), \eta(\theta)]\right|_{\theta=i \Delta \theta}\right)^{2}
$$

$$
\mathrm{E}_{m}^{\phi}=\frac{1}{\left(1+I_{k}\right)} \sum_{i=0}^{I_{k}}\left(\left.\mathrm{~N}_{2}[\phi(\theta, z)]\right|_{\theta=i \Delta \theta}\right)^{2}
$$

279 where $I_{k}$ is the number of discrete points and $\Delta \theta=\pi / I_{k}$. In this paper, $I_{k}=20$ is used.

Defining the total averaged residual square error as $\mathrm{E}_{m}^{T}=\mathrm{E}_{m}^{\varphi}+\mathrm{E}_{m}^{\eta}$, then by solving $\mathrm{dE}_{m}^{T} / \mathrm{d} c_{0}=0$, the optimal value of $c_{0}$ can be obtained, which corresponds to the minimum value of $\mathrm{E}_{m}^{T}$.

\section{Experimental Set-up}

To provide better understanding on the interaction between waves and currents and validate Key Laboratory of Hydrodynamics at Shanghai Jiao Tong University, China. The details of the facility, measurement apparatus and test conditions are described as follows.

\section{Wave Flume}

The experiments are conducted in a glass-walled wave flume $60.0 \mathrm{~m}$ long and $0.8 \mathrm{~m}$ wide with a fixed water depth of $0.5 \mathrm{~m}$. The schematic of the experimental setup is shown in Fig. 2. The flume is equipped with a hydraulically driven piston-type wave maker, while wave absorbers are equipped on the other end to absorb the incident wave energy. The following and opposing circulating currents are generated by a pump located near the wave maker.

The time series of water surface elevations are recorded by three capacitance wave gauges, which are represented by filled circles in Fig. 2. These gauges are placed along the flume with a spacing of $0.5 \mathrm{~m}$. The absolute accuracy of these wave gauges is on the order of $\pm 1 \mathrm{~mm}$. 
The duration of each record is $120 \mathrm{~s}$. The sampling frequency is $50 \mathrm{~Hz}$.

Both current and wave particle velocity measurements are made using a Nortek acoustic Doppler velocimeter (ADV) with velocity range 1m/s, sampling rate $200 \mathrm{~Hz}$, and specified accuracy of $1 \mathrm{~mm} / \mathrm{s}$. Detailed measurements of the vertical current profile and wave particle profile along the centreline of the flume are carried out.

\section{Wave-Current Condition}

The experimental conditions are listed in Table 1. The depth-averaged following and opposing current velocities are approximately $0.135 \mathrm{~m} / \mathrm{s}$ and $-0.139 \mathrm{~m} / \mathrm{s}$, respectively, in the current-only cases. Runs W1-W4 are for waves without a current, while Runs WFC1-WFC4 are waves from W1-W4 superimposed on a following current, and Runs WOC1-WOC3 are waves from W1-W3 superimposed on an opposing current. The specified wave periods input to the wave-making system for all cases are set to $1 \mathrm{~s}$, while the corresponding wave periods measured are almost constant around $1 \mathrm{~s}$. These consistent values indicate that the assumption of a constant wave period during wave-current interaction is a reasonable one for this theoretical study. On the other hand, it is noted that differences between measured wave heights and specified ones will not affect the experiment results as the measured wave heights will be used in post-processing.

In the present experiments the measuring section is located $15 \mathrm{~m}$ off the wave maker. At this location it is possible to generate the required test conditions for the duration of sufficient wave cycles. During this period regular waves coexist with the current, and the relevant experimental data are recorded before the incident wave train is disrupted by reflected waves travelling in the opposite direction. Fig. 3(a-d) shows a typical time history of the wave-only surface elevations. 
As can be seen in Fig. 3(a-d), the time histories of wave elevations for the wave-only Cases W1-W4 (around 20 to 70 seconds) appear to be quite stable, even for Cases W3 ( $a_{1} k \approx 0.25, k d \approx 2.0$ ) and W4 ( $a_{1} k \approx 0.30, k d \approx 1.9$ ), with relatively high wave steepness and low water depths. For these values of the parameters $a_{1} k$ and $k d$, Mclean (1982) pointed out that Stokes wave trains without current interaction are unstable to 3D perturbations. However, it is clear that the 3D perturbation effect in Cases W3 and W4 (around 20 to 70 seconds) is not evident. This indicates that the 3D effect in the present experiments conducted in the wave flume with the given configuration $(60 \mathrm{~m} \times 0.8 \mathrm{~m})$ is rather weak. Ma et al. (2010) also reported that experiments conducted in a wave flume with the same configuration $(60 \mathrm{~m} \times 0.8 \mathrm{~m})$ can ensure two-dimensionality of the wave field. Fig. 4(a-d) shows a typical time history comparison between wave-only cases and wave-current coexisting cases. It is worth noting that the relatively stable parts of the time histories of wave elevations for the coexisting wavecurrent Cases WFC3 ( $a_{1} k \approx 0.17, k d \approx 1.8$ ) and WOC3 ( $a_{1} k \approx 0.28, k d \approx 2.4$ ) occur around 40 to 46 seconds in Fig. 4(c). For Case WFC4 ( $\left.a_{1} k \approx 0.21, k d \approx 1.7\right)$ this relatively stable portion occurs around 20 to 26 seconds in Fig. 4(d); these stable sections were extracted from the initial phase of the complete time history of wave elevations (up to 100 seconds) for the corresponding cases as shown in Fig. 5(c1-c2, d). As seen in Fig. 5(c1-c2, d), the latter parts of the time histories of wave elevations for Cases WFC3 and WOC3 (around 55 to 95 seconds), as well as WFC4 (around 40 to 90 seconds), appear to be unstable. Since it is not the focus of the present study, no special wave gauges were arranged to obtain sufficient data to study the instability due to 3D perturbation effects in the experiment. In the present paper, the experimental measurements are used to make a comparison with the 2D HAM solution 
without any perturbation. Only the relatively stable parts of the time histories (after the initial phases) for Cases WFC3 and WOC3 (around 40 to 50 seconds), as well as Case WFC4 (around 15 to 30 seconds), as shown in the box in Fig. 5(c1-c2, d), were utilized in post-processing. These stable sections were extracted and compared to those of the wave-only Cases W3 and W4 as shown in Fig. 4(c-d). In addition, Fig. 5(a1-a2, b1-b2) was obtained from experimental measurements for a range of low wave steepness, i.e., Cases W1 ( $a k \approx 0.1, k d \approx 2.1$ ) and W2 ( $a k \approx 0.17, k d \approx 2.0$ ) with following and opposing currents; as can be seen in the figure, the complete time histories of wave elevations from 20 up to 70 seconds remain stable. This indicates that during the recording period (around 20 to 70 seconds), 3D perturbation effects are not evident for Cases W1 and W2 with following and opposing currents, i.e., Cases WFC1 ( $a k \approx 0.07, k d \approx 1.8)$, WOC1 ( $a k \approx 0.13, k d \approx 2.5)$, WFC2 $(a k \approx 0.12, k d \approx 1.7)$ and WOC2 $(a k \approx 0.24, k d \approx 2.4)$.

\section{Results and Discussion}

The analytical model, which is proposed as a solution of the interaction between nonlinear waves and a uniform current, has been validated by comparing analytical results against experimental data in the following subsection. Based on the accurate homotopy series solutions, the variation in flow characteristics due to the nonlinear interaction between steep waves and strong opposing currents is further examined in detail, together with the influence of water depth.

\section{Validation of the Analytical Model}

To validate the analytical model for nonlinear wave-current interactions, the analytical solutions are compared with the experimental measurements of wavelength and wave steepness. 
As shown in Table 2, the relative water depth of each test case in the experiment is approximately 0.3 , corresponding to an intermediate water depth condition. For the same wave period and wave height presented in Table 2, it can be seen that the wavelength and wave steepness obtained by HAM are in good agreement with the experimental data for waves with and without a current. As shown in Table 2, the experiment measured wavelength for Case W4 is approximately $1.647 \mathrm{~m}$, while the HAM obtained wavelength is about $1.640 \mathrm{~m}$. The relative error between them is $0.4 \%$. Further, Fig. 6 shows wave steepness values from the experimental data and analytical solution. It can be seen that a notable discrepancy exists for Case WOC3, i.e., the experimental data and analytical solution for wave steepness are 0.090 and 0.085 respectively. The relative error between these values is $5.6 \%$, which indicates that even for this case, the agreement between the analytical solution and the experimental data is acceptable. As shown in Table 2, the maximum total averaged residual square error $E_{m}^{T}(m=20)$ approaches the magnitude of $10^{-5}$, which further demonstrates that all the series approximation solutions are convergent and possess a high level of accuracy.

It is of interest to validate the effectiveness of the present model for the prediction of wave kinematics. The HAM solutions of horizontal velocities of water particles at the crest and trough are compared to the corresponding experimental data. In addition, the present HAM solutions and experimental measurements are also compared to numerical results obtained by the Fourier approximation method (Fenton, 1988). Fig. 7 shows the comparison of horizontal particle velocities at wave crest and trough between theoretical solutions and experiments (for cases WFC1-4 and cases WOC1-3). It can be observed that the present solutions agree well with the numerical results obtained by the Fourier approximation method. It is worth noting that the 
current distributions measured in the experiments have boundary layers near the bottom, resulting in a weak influence on the water wave dynamics (see Fig. 7). Therefore, the discrepancy in the wave kinematics near the bottom is mainly attributed to the shear current that occurs due to the bottom boundary effect. However, it will not influence the effectiveness of the present analytical model to predict wave characteristics near the free surface.

The comparisons presented above indicate that the present analytical model is capable of producing reliable predictions for nonlinear wave-current interaction in water of finite depth. In the next subsection, we will further investigate the interaction of steep waves and a strong opposing current and the influence of the opposing current and water depth on the wave characteristics.

\section{Study of Wave-Current Interaction}

To examine the influences of a strong opposing current and water depth on the free surface and wave steepness, further analytical calculations with the validated model are presented in this section, and two sets of the wave-current parameters are listed in Table 3 and Table 4, respectively.

\section{The Influence of an Opposing Current}

For a given initial wave period, the influence of an opposing current on the free surface of a nonlinear wave is considered by varying the opposing current velocities from $-0.15 \mathrm{~m} / \mathrm{s}$ to -0.4 $\mathrm{m} / \mathrm{s}$ at an interval of $0.05 \mathrm{~m} / \mathrm{s}$ (Table 3). Fig. 8 shows the free surface profiles for waves coexisting with different opposing currents at the instantaneous time of $t=0$ at a water depth of $0.5 \mathrm{~m}$, in which $a_{1}$ is set to $0.05 \mathrm{~m}$ and the wave period $T=1.01 \mathrm{~s}$ is kept constant throughout. The results are non-dimensionalised as $\eta / d$ and $x / d$. It can be observed that, 
for a given amplitude parameter $a_{1}$, the opposing current tends to narrow both the crest and corresponding trough to condense the wavelength. For example, the wavelength for the waveonly Case C1 is $1.600 \mathrm{~m}$, which is approximately 1.19 times that of the wave-current coexisting Case C2 ( $L=1.343 \mathrm{~m}$ ) with minimum opposing current velocity, and it is approximately 1.81 times that of Case C7 ( $L=0.883 \mathrm{~m}$ ) with maximum opposing current velocity. The variation in wavelength is evident, which demonstrates that an opposing current leads to a significant decrease in wavelength. Further, the elevation near the crest increases significantly while the elevation near trough appears almost unchanged. To clearly see the tendency of wave characteristics in Fig. 8, the variations in non-dimensional wavelength $L / d$, wave crest height $H_{1} / a_{1}$ and wave trough height $H_{2} / a_{1}$ are plotted against non-dimensional current velocity $\left|U_{0}\right| /(g d)^{1 / 2}$ in Fig. 9. From Fig. 9, one can see that $L / d$ decreases significantly from 3.2 to 1.7 as $\left|U_{0}\right| /(g d)^{1 / 2}$ increases from 0 to 0.18 . It is interesting to note that $H_{1} / a_{1}$ increases significantly over the range of larger current velocity values. For $\left|U_{0}\right| /(g d)^{1 / 2}$ values ranging from 0 to 0.1 , values of $H_{1} / a_{1}$ are almost constant around 1.15. As $\left|U_{0}\right| /(g d)^{1 / 2}$ increases beyond 0.1 , from 0.1 to $0.18, H_{1} / a_{1}$ increases instantly from 1.15 to 1.35 , indicating that a stronger opposing current tends to significantly increase the wave crest height. However, the corresponding wave trough height tends to remain approximately constant throughout (also see Table 3).

To further demonstrate the influence of an opposing current, wave steepness $H / L$ and the non-dimensional current velocity $\left|U_{0}\right| / C_{0}$ for Cases C1-C7 are calculated; the results are listed in Table 5 and plotted in Fig. 10, in which $C_{0}$ is the phase velocity of the linear wave at the water depth of $d=1.7 \mathrm{~m}$ which corresponds to a deep water wave condition in this paper. 
430

431

Table 5 also presents the $5^{\text {th }}$ perturbation solution of $H / L$ for the corresponding wave condition based on Fenton (1985). It can be seen that, when the opposing current velocity is low, the wave steepness values obtained by HAM and the perturbation method are almost the same. When the opposing current velocity increases, however, there exists a small discrepancy between wave steepness values $H / L$. It is worth noting that the present model can provide an estimate of the accuracy by computing the total averaged residual square errors $E_{m}^{T}$ of the $30^{\text {th }}$ HAM solution for each case as shown in Table 5. Besides, compared to the perturbation technique in Fenton (1985), the present model is much easier to apply and extend to solve more complex wave-current interaction problems. As shown in Fig. 10, one can see that at a water depth of $0.5 \mathrm{~m}, H / L$ increases up to 0.125 as $\left|U_{0}\right| / C_{0}$ increases, which indicates that the wave possesses relatively strong nonlinearity due to the effect of an opposing current. To further compare the influence of an opposing current on the wave steepness $H / L$ at different water depths, the plots of $H / L$ against the non-dimensional opposing current velocity $\left|U_{0}\right| / C_{0}$ at different water depths are also presented in Fig. 10. Again, it is seen in Fig. 10 that the $H / L$ values at each water depth increase consistently as $\left|U_{0}\right| / C_{0}$ increases. Moreover, the wave steepness increases as the water depth decreases. However, for increases in water depth beyond $0.8 \mathrm{~m}$, the wave steepness tends to be independent of the water depth. It is also observed that the water depth effect in the Cases with lower current velocity is more pronounced than that in the Cases with higher current velocity, as shown in Fig. 10. For example, for $\left|U_{0}\right| / C_{0}=0.095$, when the water depth varies from $0.4 \mathrm{~m}$ to $1.7 \mathrm{~m}$, the variation in the wave steepness is about 0.004 , which is about $5 \%$ of $H / L$ at $d=0.4 \mathrm{~m}$. However, for the maximum opposing current velocity value of $\left|U_{0}\right| / C_{0}=0.253$ in this paper, the corresponding variation in wave 
steepness is about 0.002 , which is only about $1.6 \%$ of $H / L$ at $d=0.4 \mathrm{~m}$.

Fig. 11 shows the variation of non-dimensional wavelength $L / L_{0}$ against non-dimensional opposing current velocity $\left|U_{0}\right| / C_{0}$ at different water depths $d$, in which $C_{0}$ and $L_{0}$ are the corresponding phase velocity and wavelength of the linear wave at a water depth of $d=1.7$ m. It is clearly seen that at each water depth $L / L_{0}$ decreases as $\left|U_{0}\right| / C_{0}$ increases. With the same current strength and water depths ranging from $0.4 \mathrm{~m}$ to $0.8 \mathrm{~m}$, a decrease in water depth also leads to a decrease in wavelength; however, for water depths beyond $0.8 \mathrm{~m}$, the wavelength tends to be independent of the water depth. It is important to note that the water depth effect on $L / L_{0}$ also dominates over the range of lower opposing current velocities and the value of $L / L_{0}$ for each water depth tends to approach an identical value as $U_{0} / C_{0}$ increases. For instance, for $\left|U_{0}\right| / C_{0}=0.095$, when the water depth varies from $0.4 \mathrm{~m}$ to $1.7 \mathrm{~m}$, the variation in $L / L_{0}$ is about 0.038 , which is about $4.6 \%$ of $L / L_{0}$ at $d=0.4 \mathrm{~m}$. However, for the maximum opposing current velocity $\left|U_{0}\right| / C_{0}=0.253$, the corresponding variation in $L / L_{0}$ is about 0.003 , which is only about $0.57 \%$ of $L / L_{0}$ at $d=0.4 \mathrm{~m}$. It is clearly seen that, as the opposing current velocity increases, the percent variation in the non-dimensional wavelength due to changes in water depth is much smaller than variations in wave steepness. That is why the value of $L / L_{0}$ for each water depth tends to approach an asymptotic value (about 0.55) as $\left|U_{0}\right| / C_{0}$ increases.

\section{The Influence of Water Depth}

To investigate how decreases in water depth influence wave steepness for the case of waves coexisting with an opposing current, the initial wave period is also kept constant throughout. Then the influence of water depth on the wave steepness under different opposing current 
velocities is considered by varying the water depth from $0.1 \mathrm{~m}$ to $0.7 \mathrm{~m}$ at an interval of $0.1 \mathrm{~m}$ (for larger water depths) and $0.05 \mathrm{~m}$ (for smaller water depths) as shown for $U_{0}=-0.3 \mathrm{~m} / \mathrm{s}$ in Table 4. For a constant wave period (0.76 s), the wavelengths presented in Table 4 are used as the input to the present model. From Table 4, it is clearly seen that for water depths ranging from $0.1 \mathrm{~m}$ to $0.3 \mathrm{~m}$, the variation in water depth leads to a relatively significant variation in wavelength. However, for water depths beyond $0.3 \mathrm{~m}$, the variation in water depth only results in small variations in wavelength. This is a further demonstration that the water depth has an evident effect on the wavelength starting from an intermediate water depth condition.

The HAM solutions for wave steepness $H$ / $L$ for Cases D1-D9 are plotted against relative water depth $d / L$ in Fig. 12, and the values are listed in Table 6 with the corresponding total averaged residual square errors $\mathrm{E}_{m}^{T}$. As shown in Table 6, for $d / L<0.5$, a decrease in $d / L$ leads to a distinct increment in $H / L$, while for $d / L>0.5$ (which corresponds to a deepwater wave condition in the present study), the influence of the variation in $d / L$ on the $H / L$ is less evident. On the other hand, although the value of $\mathrm{E}_{m}^{T}$ for Case D9 is relatively high $\left(6.360 \times 10^{-3}\right)$, it is noted that the nonlinearity in this case is very high resulting from a stronger opposing current and shallower water depth. To further investigate the influence of water depth on wave steepness under different opposing current velocities, additional calculations are carried out and the results are plotted in Fig. 12. It can be seen in Fig. 12 that for each opposing current, a transitional point exists and divides the curve into two parts. By connecting all the transitional points, it is clearly seen that on the left side of the transition line, the values of $H / L$ increase significantly as $d / L$ decreases. On the right side of the transition line, however, $H / L$ is independent of $d / L$. As shown in Fig. 12, $H / L$ 
496

497

498

499

500

501

502

503

504

505

506

507

508

509

510

511

512

513

514

515

increases to a higher value (approximately 0.12 ) as the opposing current velocity increases. This is a further demonstration of the influence of the opposing current on the wave steepness.

\section{Conclusion}

In this paper, an analytical approximation of nonlinear wave-current interaction in water of finite depth is derived using the homotopy analysis method. Series approximation solutions are obtained and compared to experimental and available numerical results; they demonstrate that the present method not only gives highly accurate results of wave parameters for the interaction between steep waves and a strong opposing current, but the method also produces excellent results for wave kinematics. Based on the validated analytical model, the interaction between waves and a strong opposing current is investigated to clarify the influence of the strong opposing current and water depth on the wave profile, wavelength and wave steepness.

The following key conclusions can be drawn from the present study:

(1) The accuracy and convergence of the series approximation solutions obtained by the proposed method are verified by estimating the errors of the exact kinematic and dynamic free surface boundary conditions, and by comparing the present experimental measurements and to an available numerical solution. This demonstrates the proposed homotopy analysis method is a very effective technique to study nonlinear waves interacting with a strong current in finite water depths.

(2) An opposing current leads to significant decreases in wavelength and tends to narrow both the crest and trough. The wave crest elevation increases as the opposing current velocity increases, and the wave trough elevation tends to remain constant throughout.

(3) The wave steepness $H / L$ at each water depth increases consistently as the non- 
dimensional opposing current velocity $\left|U_{0}\right| / C_{0}$ increases. It is also observed that the water depth effect in the case of smaller opposing current velocity is more pronounced than that in the case of larger opposing current velocity.

(4) At each water depth the non-dimensional wavelength $L / L_{0}$ decreases as the nondimensional opposing current velocity $\left|U_{0}\right| / C_{0}$ increases. The water depth effect on $L / L_{0}$ dominates over the range of smaller opposing current velocities, and the value of $L / L_{0}$ for each water depth tends to approach an asymptotic value as $U_{0} / C_{0}$ increases.

(5) Under the existence of an opposing current, a decrease in relative water depth $d / L$ leads to an increase in wave steepness $H / L$. Two regimes exist and are separated by a transition line: on the left side of the transition line, the value of $H / L$ increases significantly as $d / L$ decreases, while on the right side of the transition line, the value of $H / L$ is independent of $d / L$.

The method presented in this paper can be applied to solve more complex scenarios of nonlinear wave interaction with strong currents in water of finite depths leading to engineering applications in the coastal and offshore industries.

\section{Acknowledgments}

The authors would like to express their gratitude to the National Natural Science Foundation of China (Grant No.51239007, 51209136) and Newton Research Collaboration Programme Award, The Royal Academy of Engineering for financial support. The authors would also like to express their thanks to Prof. Hua Liu and Prof. Yongliu Fang for their assistance during the experiments.

\section{References:}

Chen, Y.-Y., and Chen, H.-S. (2014). “Lagrangian solution for irrotational progressive water 
waves propagating on a uniform current: Part 1. Fifth-order analysis.” Ocean Eng., 88, 546567.

Cheng, J., Cang, J., and Liao, S. J. (2009). "On the interaction of deep water waves and exponential shear current ” Z. Angew. Math. Phys., 60, 450-478.

Fenton, J. D. (1985). “A fifth-order stokes theory for steady waves.” J. Waterway, Port, Coastal, Ocean Eng., 10.1061/(ASCE)0733-950X(1985)111:2(216), 216-234.

Fenton, J. D. (1988). "The numerical solution of steady water wave problems.” Computers and Geosciences, 14 (3), 357-368.

Jonsson, I. G. (1990). “Wave-current interactions.” In The Sea, Ocean Eng. Science 9A, Eds. B. Le Mehaute and D. M. Hanes, 65-120.

Kharif, C., and Pelinovsky, E. (2003). "Physical mechanisms of the rogue wave phenomenon.” Eur. J. Mech. B-Fluid., 22, 603-634

Liao, S. J. (2003). Beyond Perturbation: Introduction to the Homotopy Analysis Method. Chapman \& Hall/CRC, Florida.

Liao, S. J. (2011). “On the homotopy multiple-variable method and its applications in the interactions of nonlinear gravity waves.” Commun. Nonlinear Sci. Numer. Simul., 16, 12741303.

Liao, S. J., and Cheung, K. F. (2003). "Homotopy analysis of nonlinear progressive waves in deep water.” J. Eng. Math., 45(2), 105-116.

Lin, Z., Tao, L., Pu, Y., and Murphy, A. (2014). “Fully nonlinear solution of bi-chromatic deepwater waves.” Ocean Eng., 91, 290-299.

Liu, Z., and Liao, S. J. (2014). "Steady-state resonance of multiple wave interactions in deep 
water.” J. Fluid Mech., 742, 664-700.

Liu, Z., Lin, Z., and Liao, S. J. (2014). "Phase velocity effects of the wave interaction with exponentially sheared current.” Wave Motion, 51, 967-985.

Ma, Y., Dong, G., Perlin, M., Ma, X., Wang, G., and Xu, J. (2010). “Laboratory observations of wave evolution, modulation and blocking due to spatially varying opposing currents.” J. Fluid Mech., 661, 108-129.

Mallory, J. K. (1974). “Abnormal waves in the south-east coast of South Africa.” Int. Hydrog. Rev., 51, 99-129.

McLean, J. W. (1982). “Instabilities of finite-amplitude gravity waves on water of finite depth.” J. Fluid Mech., 114, 331-341.

Peregrine, D. H. (1976). “Interaction of water waves and currents.” Adv. Appl. Mech. 16, 9-117. Rienecker, M. M., and Fenton, J. D. (1981). “A Fourier approximation method for steady water waves.” J. Fluid Mech., 104, 119-137.

Swan, C., Cummins, I. P., and James, R. L. (2001). “An experimental study of two-dimensional surface water waves propagating on depth-varying currents: Part 1. Regular waves” J. Fluid Mech., 428, 273-304

Swan, C., and James, R. L. (2001). “A simple analytical model for surface water waves on a depth-varying current.” Appl. Ocean Res., 22, 331-347.

Tao, L., Song, H., and Chakrabarti, S. (2007). "Nonlinear progressive waves in water of finite depth-an analytic approximation.” Coast. Eng., 54, 825-834.

Thomas, G. P. (1981). “Wave-current interactions: an experimental and numerical study: Part 1. Linear waves” J. Fluid Mech., 110, 457-474. 
584 Thomas, G. P. (1990) "Wave-current interactions: an experimental and numerical study: Part 2.

585 Nonlinear waves” J. Fluid Mech., 216, 505-536.

586 Thomas, G. P., and Klopman, G. (1997). "Wave-current interactions in the nearshore region.” 587 In Gravity Waves in Water of Finite Depth, Ed. J. N. Hunt, 215-319.

588 Umeyama, M. (2011). "Coupled PIV and PTV measurements of particle velocities and 589 trajectories for surface waves following a steady current.” J. Waterway, Port, Coastal, Ocean $590 \quad$ Eng., 10.1061/(ASCE)WW.1943-5460.0000067, 85-94.

591 Xu, D., Lin, Z., Liao, S., and Stiassnie, M. (2012). “On the steady-state fully resonant 592 progressive waves in water of finite depth.” J. Fluid Mech., 710, 379-418.

$593 \mathrm{Xu}, \mathrm{H}$. (2006). Applications of the homotopy analysis method in fluid mechanics and ocean 594 engineering. PhD thesis, Shanghai Jiao Tong University. 
Fig. 1. Definition sketch

598

599

600

601

602

603

604

605

606

607

608

609

610

611

612

613

614

615

616

617

Fig. 2. Schematic of experimental setup (a) Elevation view (b) Plan view

Fig. 3. Time history of surface elevation for wave-only Case W1 W4 (Recorded with wave gauge 1)

Fig. 4. Time history of surface elevation (recorded with wave gauge 1): comparison between wavesonly cases and wave-current coexisting cases (W: waves-only; FC: following current, $U_{0}=0.135 \mathrm{~m} / \mathrm{s}$;

OC: opposing current, $U_{0}=-0.139 \mathrm{~m} / \mathrm{s}$ ).(a) W1: $T=1.000 \mathrm{~s}, \quad H=0.046 \mathrm{~m}$; WFC1: $T=0.999 \mathrm{~s}$, $H=0.037 \mathrm{~m}$; WOC1: $T=1.002 \mathrm{~s}, \quad H=0.053 \mathrm{~m}$; (b) W2: $T=1.000 \mathrm{~s}, \quad H=0.086 \mathrm{~m}$; WFC2: $T=1.002 \mathrm{~s}$, $H=0.068 \mathrm{~m}$; WOC2: $T=1.001 \mathrm{~s}, \quad H=0.100 \mathrm{~m}$; (c) W3: $T=1.000 \mathrm{~s}, \quad H=0.124 \mathrm{~m}$; WFC3: $T=1.001 \mathrm{~s}$, $H=0.098 \mathrm{~m}$; WOC3: $T=1.002 \mathrm{~s}, \quad H=0.116 \mathrm{~m}$; (d) W4: $T=1.003 \mathrm{~s}, \quad H=0.149 \mathrm{~m}$; WFC4: $T=1.001 \mathrm{~s}$, $H=0.120 \mathrm{~m}$.

Fig. 5. Time history of surface elevation for wave-current coexisting Case WFC1 WFC4 and WOC1 WOC3 (Recorded with wave gauge 1)

Fig. 6. Wave steepness comparison between HAM solution and experimental data

Fig. 7. Horizontal fluid velocity under the wave crest and trough: comparison between the present HAM solutions, the present experimental results and the Fourier approximation of Fenton (1988) ( $z=0$ at seabed)

Fig. 8. Wave profile comparison between waves with different current velocity at $\mathrm{t}=0$ ( $d=0.5 \mathrm{~m}$, $\left.a_{1}=0.05 \mathrm{~m}, \quad T=1.01 \mathrm{~s}\right)$

Fig. 9. Variation of non-dimensional wavelength, wave crest height and wave trough height against non-dimensional opposing current velocity ( $d=0.5 \mathrm{~m}, a_{1}=0.05 \mathrm{~m}, T=1.01 \mathrm{~s}$ )

Fig. 10. Variation of wave steepness against the non-dimensional opposing current velocity at different 

water depth $\left(a_{1}=0.05 \mathrm{~m}, T=1.01 \mathrm{~s}\right)$

619 Fig. 11. Variation of non-dimensional wavelength against non-dimensional opposing current velocity at 620 different water depth $\left(a_{1}=0.05 \mathrm{~m}, T=1.01 \mathrm{~s}\right)$

621 Fig. 12. Variation of wave steepness against relative water depth at different current velocity 
Table 1. Wave-Current Conditions

Table 2. Comparison of 20-order HAM Approximations of Wavelength $L$ and Wave Steepness $H / L$

627 with Experimental Data

628 Table 3. Wave-Current Parameters for Current Velocity Parametric Study ( $d=0.5 \mathrm{~m}, a_{1}=0.05 \mathrm{~m}$,

$629 T=1.01 \mathrm{~s})$

630 Table 4. Wave-Current Parameters for Water Depth Parametric Study ( $U_{0}=-0.3 \mathrm{~m} / \mathrm{s}, \quad a_{1}=0.021 \mathrm{~m}$,

631 $T=0.76 \mathrm{~s})$

632

Table 5. The 30-order HAM Approximations of Wave Steepness $H / L$ against Non-dimensional

633 Current Velocity $U_{0} / C_{0}$ together with $\mathrm{E}_{m}^{T}\left(d=0.5 \mathrm{~m}, a_{1}=0.05 \mathrm{~m}, T=1.01 \mathrm{~s}\right)$

634 Table 6. The 30-order HAM Approximations of Wave Steepness $H / L$ against Relative Water Depth 635 $d / L$ together with $\mathrm{E}_{m}^{T} \quad\left(U_{0}=-0.3 \mathrm{~m} / \mathrm{s}, \quad a_{1}=0.021 \mathrm{~m}, T=0.76 \mathrm{~s}\right)$ 